\title{
Numerical Simulation of Double Surface Liquid Ejector with Flow Swirl for Centrifugal Pump
}

\author{
A. A. Lyamasov ${ }^{1 *}$, B. M. Orahelashvili ${ }^{1}$ and A. M. Gribkov ${ }^{1}$ \\ ${ }^{1}$ MPEI National Research University, Department of hydromechanics and hydraulic machines, \\ Moscow, Russia
}

\begin{abstract}
Many systems with liquid ejectors and centrifugal pump are known. Often, jet pumps are used to provide a self-priming mode, as well as an acceptable pressure level for cavitation-free operation. The main disadvantage of such systems is the relatively low efficiency associated with the peculiarities of energy transfer in ejector. To increase efficiency double surface jet pump with driving and suction flow swirl (with circumferential component of velocity) is proposed. The active flow swirling is ensured by using of multi-nozzle tangential nozzle inlet and passive flow part by a special blade system. Combination of these factors makes it possible to improve the efficiency of energy conversion process. In comparison with the known design increases pump efficiency by $10 \%-15 \%$. Flow swirl also permits to reduce horizontal overall size by increasing the diffuser angle and reducing the mixing chamber length. These positive effects can be achieved by using methods and recommendations given in this paper. The paper also includes ANSYS CFX numerical simulation study results of double surface jet pump and analysis of the impact of nozzle position, length of the mixing chamber and other geometry parameters on pump performance. The results allow optimize the constructive solutions.
\end{abstract}

\section{Introduction}

Jet pumps (JP) are fluidic apparatuses that use for pumping energy of external liquids, gases or vapours. These hydraulic machines are widely used in many areas due to several their significant advantages over other types of pumping equipment:

- high reliability due to the absence of moving parts;

- possibility of self-priming;

- minimum sensitivity of work process to gas and solids;

- life-time is limited only by the properties of the materials used in the manufacture;

- low material consumption, compactness, (can be placed without special fastening and foundations);

- easy pump starting (absolutely safe against dry running) and stopping.

Often, jet pumps are used to provide a self-priming mode, as well as an acceptable pressure level for cavitation-free operation.

* Corresponding author: a-lyamasov@mail.ru 
Despite these advantages, jet pumps have a significant drawback - low energy efficiency, not more than 35-40\%. Regardless of design features of liquid ejector they use direct energy transfer from one flow with its large level to another (the first one is driving, and the second is suction flow). The quality of the process of energy transfer from driving to suction flow in a jet pump directly affects its efficiency. As a result, to improve the efficiency a process of energy exchange between flows should be improved. The ways to improve the interaction of flows and reduce losses in the elements of JP include:

- improving JP flow parts geometry;

- use of new materials and special coatings;

- flow swirl;

- transition from stationary driving flow ejection to non-stationary (pulsating flow JP; JP with rotating nozzle or mixing chamber; JP with a change in the direction of driving flow etc.).

The first two ways are currently intensively studied for known and common jet pumps: with a round central nozzle and an annular single surface nozzle. We consider that usage of a central double surface nozzle may be a promising way to improve geometry for increasing JP efficiency.

The last two ways have not been studied enough at present and there are no unified theories that explain them. Moreover, the technical implementation of non-stationary flow principles in a JP is often accompanied by a loss of operational reliability due to the introduction of moving parts or strong influence on external structures (vibration, temperature variations etc.).

Based on the analysis, as well as using the principles of the Theory of Invention Tasks Solving (TRIZ), a promising JP design was developed, which combines two methods for improving energy transfer efficiency - an interacting flows swirl and a double-surface annular nozzle.

\section{Structural synthesis}

\subsection{Double surface nozzle}

Energy is transferred from the driving to the suction flow in JP at the interaction surfaces and therefore these hydraulic machines are also known as frictional pumps. Today, the most common are designs with a central round nozzle and an annular nozzle (figure 1, (a) and (b)). In such pumps there is one surface of flows interaction. Pump with central circular nozzle creates one interaction surface which is designated on figure 1 (a) by digit 1 and a pump with an annular nozzle also creates one interaction surface which is designated on figure 1 (b) by digit 2. A pump with a central annular nozzle shown on figure 1 (c) is characterized by two surfaces of flow interaction -1 and 2 . This contributes to the most efficient transfer of energy from driving to suction flow, because area of their interaction increases.

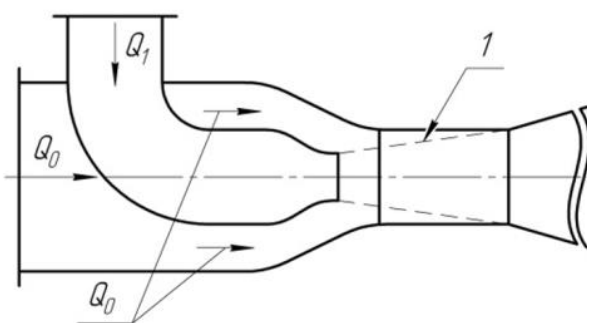

(a)

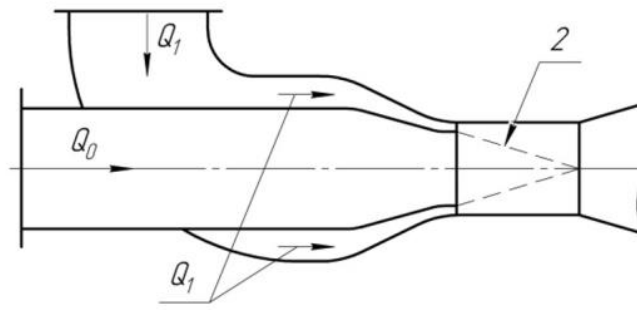

(b) 


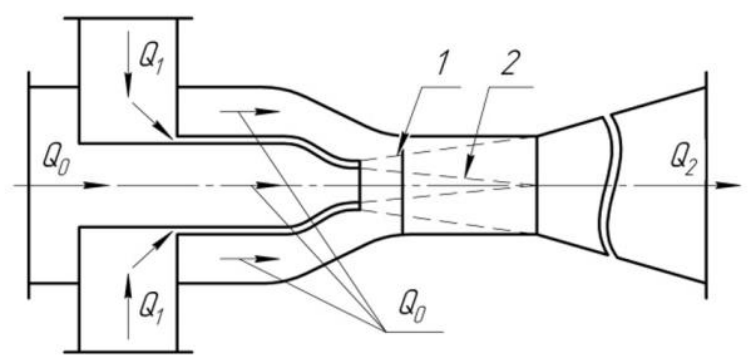

(c)

Fig. 1. Flow interaction surfaces for various types of nozzles: central round nozzle (a); annular nozzle (b); central annular nozzle (c).

\subsection{Flow swirl}

An essential prerequisite for the use of swirling flows in JP is their property to increase the attached mass (suction flow discharge) compared to flows without circumferential component of velocity [1], [3]. This effect is shown in figure 2 for round submerged flow.

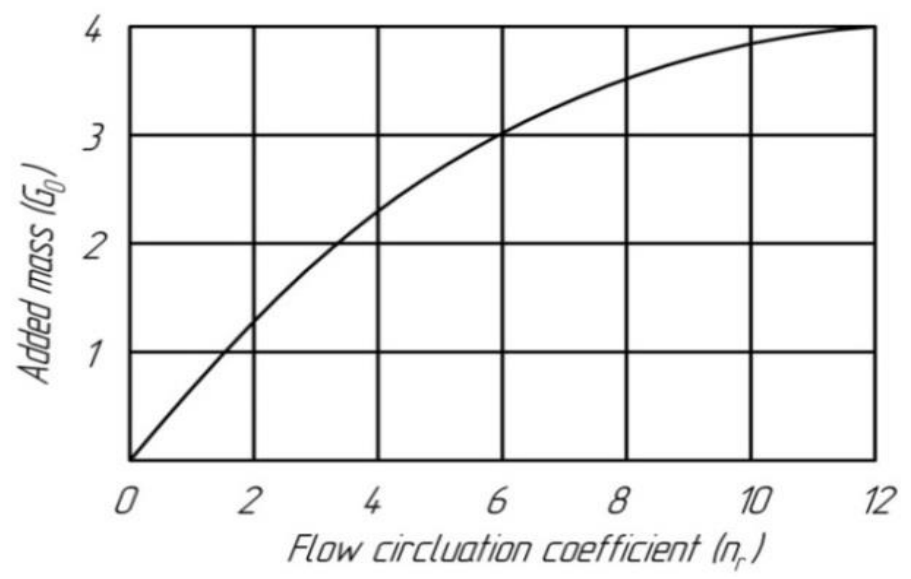

Fig. 2. Effect of flow swirl on added mass for round submerged flow.

Circumferential component of velocity at the nozzle exit can be obtained in different ways: by special inlets of the driving flow (spiral, tangential, multi-channel), by blade systems, cuts and ribs, etc. As a result of the analysis of various methods, it was found that the most effective way of swirling the submerged annular flow is a multi-channel tangential nozzle inlet. Preliminary numerical simulations showed that this method makes it possible to get the necessary flow swirl without destroying its structure. Moreover, the changing of channels angle allows you to influence the value of the circumferential component of velocity.

In the same axial distance flow swirl increases the length of the interacting flows streamlines. This is a contributing factor in increasing of JP efficiency.

In the liquid ejector with flow swirl the mixing flow at the diffuser is pressed against the walls due to circumferential effect. This allows increasing of diffuser efficiency due to angle increasing and allows reducing the length of the diffuser and the dimensions of the JP as a whole. 


\subsection{New type jet pump design}

The double surface JP with flow swirl design is shown in figure 3 and figure 4.

The double surface annular nozzle 4 is located in the central part of the suction chamber 2. The liquid is supplied to the nozzle through the tangential channels 1 , which are located inside the stationary blades 5 . The blades 5 are located between the wall of the suction chamber 2 and the annular nozzle 4 . They simultaneously perform several functions: drive flow inlet, integrate and strengthen the structure, reduce hydraulic losses of suction flow (compared to other nozzle installation methods) and swirl part of suction flow.

Suction and driving flows are interacting in cylindrical mixing chamber 3 . In the diffuser 6 kinetic energy of the mixed flow is converted into static pressure.

As shown in figure 3 ejector geometry at the nozzle exit can be identified by three flows areas:

1) Suction annular flow swirled by blades of guiding apparatus $\left(D_{0}-d_{1}\right)$;

2) Driving annular flow swirling by tangential channels $\left(d_{0}{ }^{\prime \prime}-d_{0}{ }^{\prime}\right)$;

3) Suction cylindrical flow $\left(d_{0}\right)$.

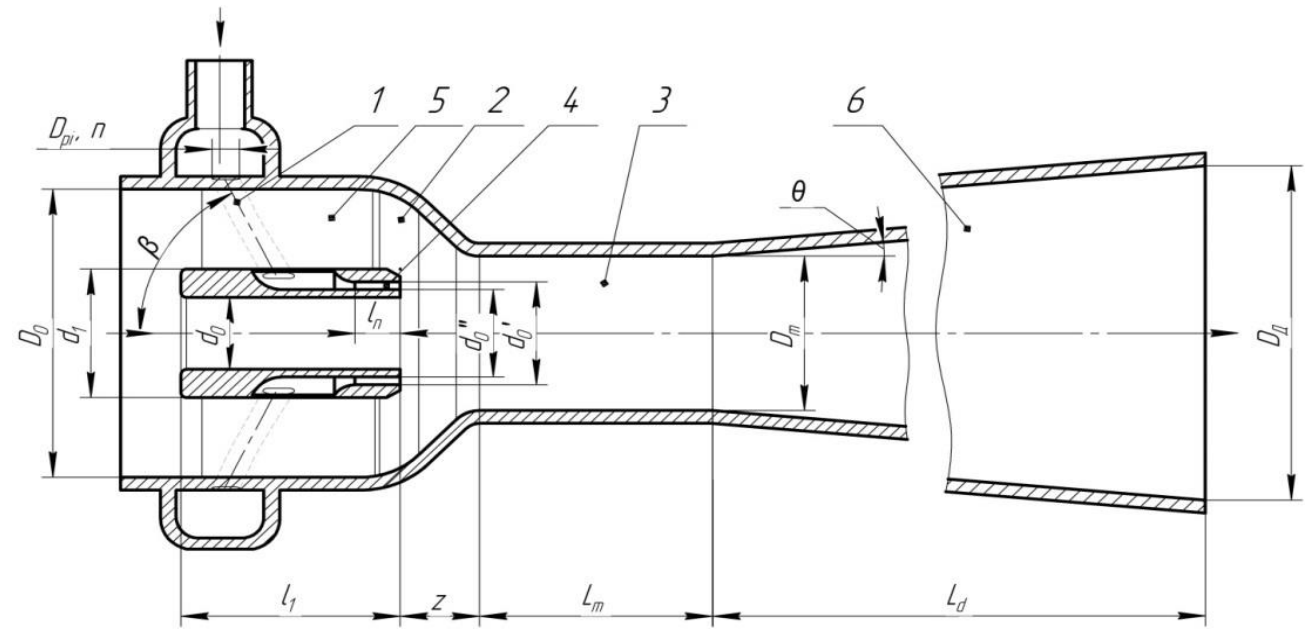

Fig. 3. New type double surface jet pump design: $(1-$ tangential channels, 2 - suction chamber, 3 - mixing chamber, 4 - annular nozzle, 5 - blades (guiding apparatus), 6 - diffuser).
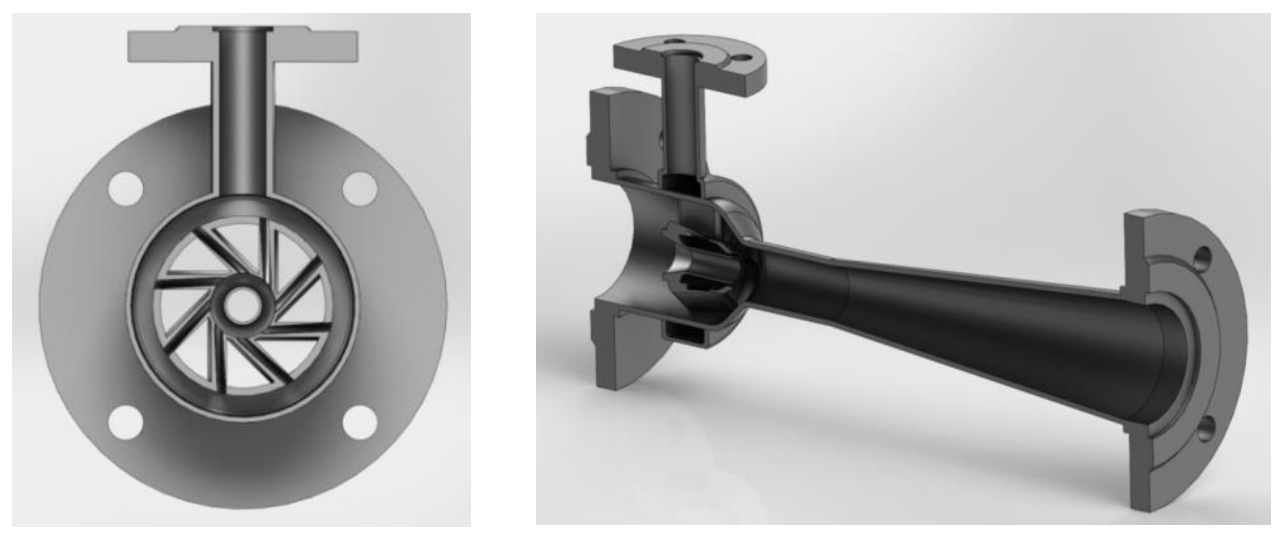

Fig. 4. New type jet pump cross and longitudinal cutaway views. 


\section{JP dimensions and main characteristics}

Initial parameters for proposed methodology of double surface JP with flow swirl design are the following - head ratio $\mathrm{h}$ and mixed flow rate $Q_{2}$. For this pump efficiency increase corresponds to the increase of flow ratio $q$ :

$$
\begin{aligned}
& h=\frac{H_{s}}{H_{s}+H_{d}}, \\
& Q_{2}=Q_{0}+Q_{1}, q=\frac{Q_{0}}{Q_{1}} .
\end{aligned}
$$

As shown in figure $1 Q_{0}$ is a suction flow rate, and $Q_{1}$ is a driving flow rate.

The first approximation of the JP with an annular nozzle geometry characteristics is calculated using the following equations:

$$
\begin{aligned}
& q=\frac{k}{1-\varphi_{d}^{2} \varphi_{i n}^{2}}\left(\sqrt{1+\left(m \varphi_{d}^{2}-1\right)\left(1-\varphi_{d}^{2} \varphi_{i n}^{2}\right)}-1\right) \\
& m=(1+q)^{2}\left(1+\zeta_{d}\right)-\frac{q^{2}}{1+\zeta_{i n}} \\
& v_{n}=\varphi_{n} \sqrt{2 g H_{d}} \\
& F_{n}=\frac{Q_{1}}{v_{n}}, F_{m c}=m F_{n}
\end{aligned}
$$

In equations (3)-(6) $k$ - correction coefficient for annular nozzle; $m$ - the mixing chamber $F_{\mathrm{mc}}$ and annular nozzle $F_{\mathrm{n}}$ areas ratio; $\varphi_{\mathrm{n}}, \varphi_{\mathrm{d}}, \varphi_{\mathrm{in}}-$ nozzle, diffuser and inlet velocity coefficients; $\zeta_{\mathrm{d}}, \zeta_{\text {in }}-$ diffuser and inlet hydraulic loss coefficients.

Further research of the JP showed the need to adjust the geometric parameters obtained by the above equations to achieve a higher efficiency. Particularly, correction coefficient for annular nozzle should be clarified.

\section{Numerical simulation of the JP working process}

For this research an approach of numerical modeling of hydrodynamic processes was used. A high-performance computational fluid dynamics (CFD) software ANSYS CFX is used for this purpose. In the future, based on the obtained data, it is planned to refine the mathematical model written on the basis of equations (1) and (2). This in turn will make it possible to refine equations (3) and (4) for the design of double surface JP with flow swirl.

The numerical simulation was carried out according to the following stages (figure 5):

- creating a geometric model of the flow part;

- creating a mesh control volumes on the model of the flow part (domain);

- setting boundary conditions;

- numerical solution based on k-e turbulence model;

- analysis of the results. 


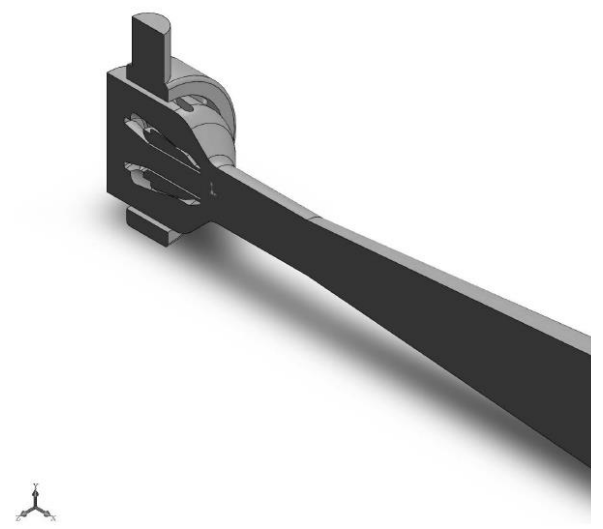

(a)

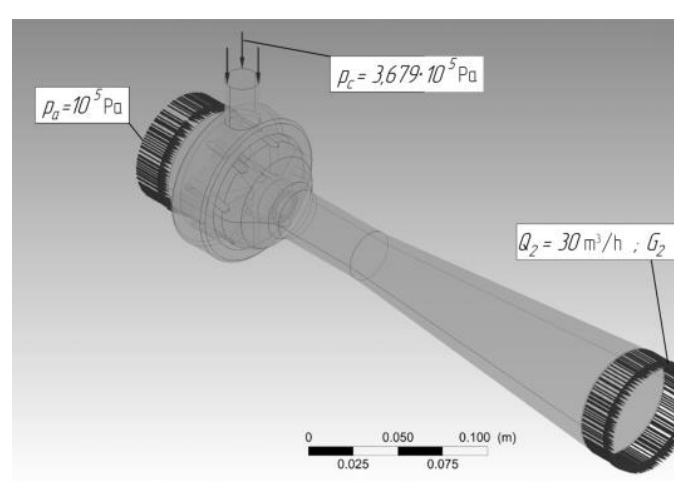

(b)

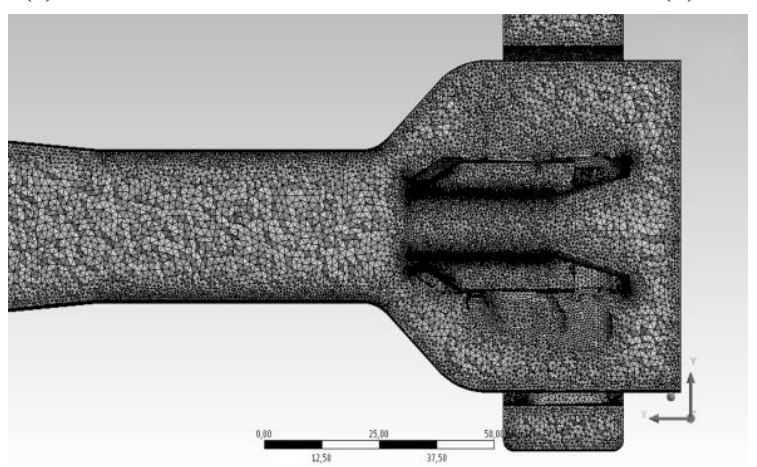

(c)

Fig. 5. Calculation domain (a), boundary conditions (b) and mesh control volumes (c).

In the mesh generating process three types of elements were used: tetrahedral (Tet4), prismatic (Wed6) and pyramids (Pyr5). Some mesh parameters are presented in tables 1 and 2.

Table 1. Some parameters of the mesh control volumes.

\begin{tabular}{|c|c|}
\hline Parameter name & Value \\
\hline Max Face Size (mm) & 1.2 \\
\hline Max Tet Size (mm) & 3.0 \\
\hline Curvature Normal Angle (deg) & 18 \\
\hline Growth Rate & 1.2 \\
\hline Maximum thickness ${ }^{*}(\mathrm{~mm})$ & 0.7 \\
\hline Number of layers ${ }^{*}(\mathrm{~mm})$ & 6 \\
\hline Growth Rate* & 1.2 \\
\hline
\end{tabular}

Table 2. Some parameters of the mesh control volumes.

\begin{tabular}{|l|l|}
\hline Parameter name & Value \\
\hline
\end{tabular}




\begin{tabular}{|c|c|}
\hline Nodes & 1064996 \\
\hline Elements & 3322101 \\
\hline $\begin{array}{c}\text { Orthogonality quality } \\
\text { (Min/Max/Average) }\end{array}$ & $0,14381 / 0,99998 / 0,86576$ \\
\hline $\begin{array}{c}\text { Slanting } \\
\text { (Min/Max/Average) }\end{array}$ & $6,2128 \mathrm{e}-6 / 0,89214 / 0,23528$ \\
\hline Aspect ratio (Min/Max/Average) & $1,1595 / 37,706 / 4,3636$ \\
\hline Y+Max & 201.2 \\
\hline
\end{tabular}

Due to the small area of the nozzle, the annular volume and the areas near it are divided by a denser mesh to increase the accuracy of the calculation. Also, the mesh is condensed in the area of the flow distribution (the suction chamber and the mixing chamber volumes).

\section{Results and discussion}

According to the previously presented algorithm, three types of JP were investigated in ANSIS CFX:

- With central round nozzle (figure 1, a). These pumps are widely used, and their characteristics are well known. Analysis of the characteristics of such an ejector allows us to draw conclusions about the reliability off the results, and a comparison with these characteristics allows us to draw conclusions about the efficiency of the pump with a flow swirl. The pump is designed according to the methodology of Sokolov and Zinger [4].

- With central annular nozzle (double surface) without flow swirl. Analysis of the characteristics of such an ejector allows us to draw conclusions about double surface JP. The pump is designed according to the methodology of Aleksandrov and Tarasiyanc [5], [8].

- With central annular nozzle (double surface) with flow swirl.

Jet pumps have been designed with the following operating parameters for work in pare with $4 \mathrm{~K}-12$ centrifugal pump according of the assemble on figure 6 :

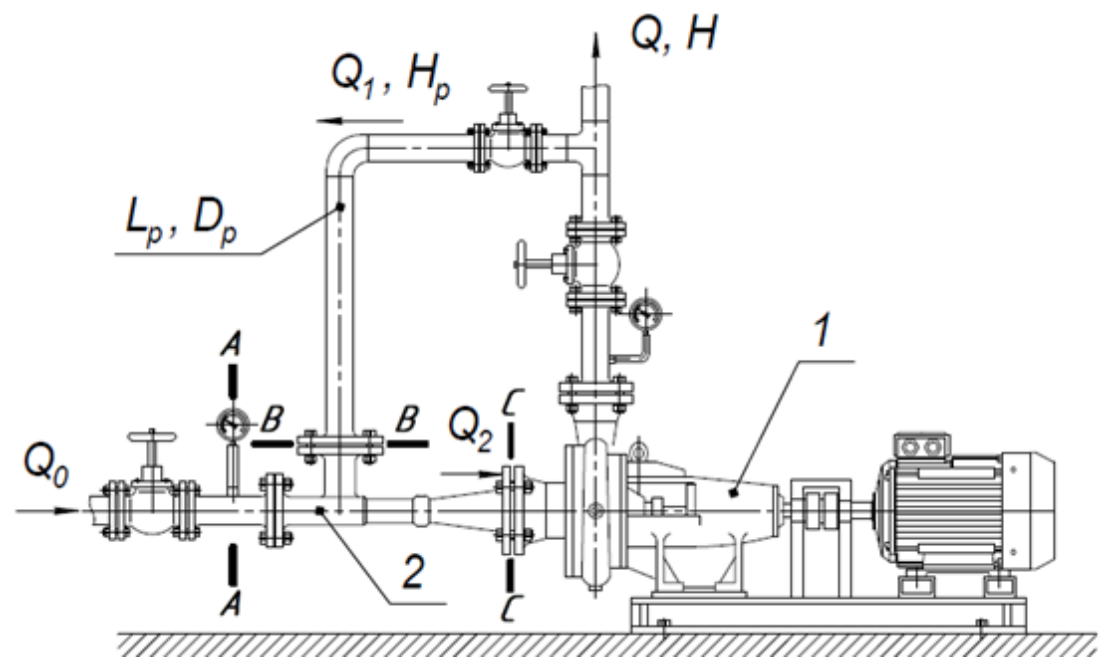

Fig. 6. Centrifugal pump 4K-12 (1) with JP (2).

The parameters of the pump-ejector unit are the following:

- head ratio $h=0.074\left(H_{s}=3.0 \mathrm{~m}, H_{d}=37.5 \mathrm{~m}\right)$; 
- mixed flow rate $Q_{2}=30 \mathrm{~m}^{3} / \mathrm{h}$.

Dependences $h(q)$ and $\eta(q)$ were obtained based on the numerical modelling in ANSYS CFX (figure 7).

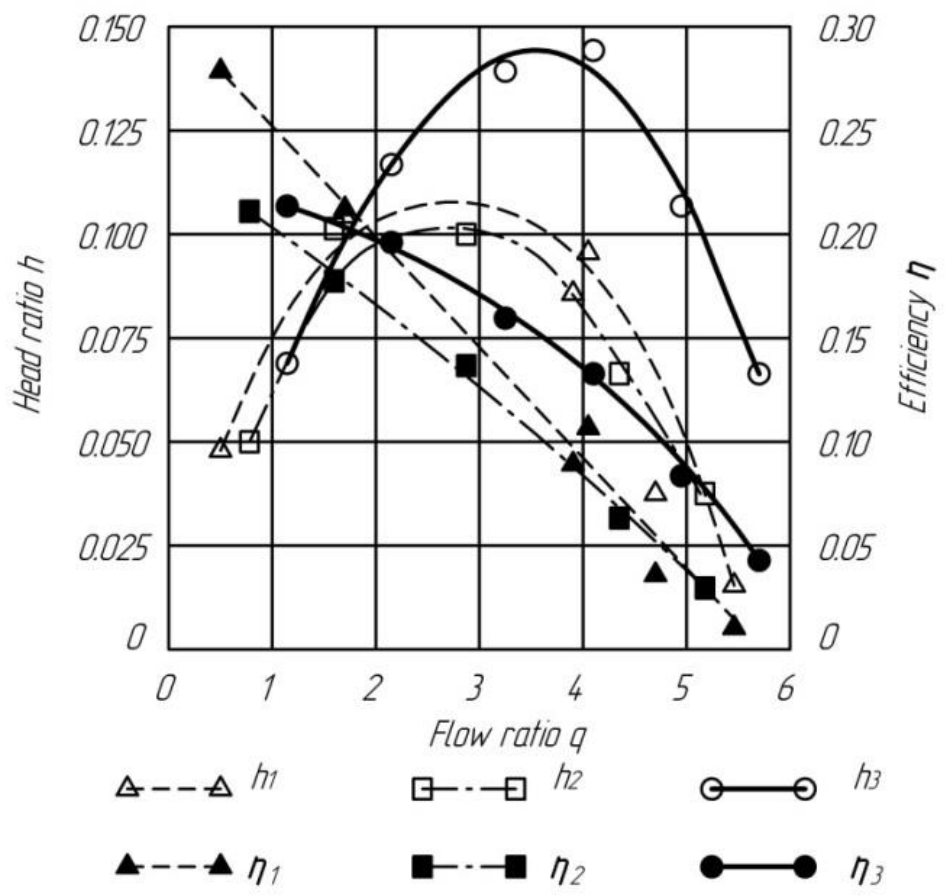

Fig. 7. Different JP types curves.

Index "1" is used for JP with central round nozzle, "2" for JP with central annular nozzle (double surface) without flow swirl and "3" for JP with central annular nozzle (double surface) with flow swirl.

The curves of ejector with central round nozzle (figure 6, "1") allow us to accept calculation accuracy because they are close to theoretical values (based on the experimental data).

The study of the curves for the JP with central annular nozzle without flow swirl (figure 6 , "2") showed that the existing design methodology [5] does not allow obtaining the desired result of efficiency. Analysis of the speed and pressure vectors in the flow part of the pump allowed to conclude that the main reason of low efficiency is incorrect recommended ratio of the annular nozzle and the mixing chamber diameters. The methodology was developed for other values of the q, which may be the cause of these discrepancies. Suction annular flow (figure 3 ) in that case was almost completely clamped and did not make the desired impact to transfer of energy from driving to suction flow. This effect has been taken into account and the geometry of JP with central annular nozzle (double surface) with flow swirl has been adjusted. Curves " 3 " were obtained.

Table 3. Comparison of various types of jet pumps.

\begin{tabular}{|c|c|c|c|}
\hline Jet pump type & $\eta$ & $q$ & $L, \mathrm{~mm}$ \\
\hline With central round nozzle & 0,21 & 3,0 & 495 \\
\hline
\end{tabular}




\begin{tabular}{|c|c|c|c|}
\hline $\begin{array}{c}\text { With central annular nozzle } \\
\text { (double surface) without flow } \\
\text { swirl }\end{array}$ & 0,20 & 2,5 & 350 \\
\hline $\begin{array}{c}\text { With central annular nozzle } \\
\text { (double surface) with flow } \\
\text { swirl }\end{array}$ & 0,29 & 3,7 & 260 \\
\hline
\end{tabular}

The curves show an increase of flow ratio $q$ in the version with flow swirl, which is confirmed by experimental data (figure 2). In JP designed for the same parameters, the values of efficiency raised up to 0.29 , which significantly exceeds the pump without swirling and with a central nozzle. Some main parameters of the JP with a central nozzle, a two-surface ejector with and without swirl are summarized in table 3.

The calculations in ANSYS CFX, as well as preliminary engineering design prove the advantages of a double-surface JP with a flow swirl: increase of efficiency and reduction of overall length. In this regard, numerical modelling was performed to study the effect of JP various geometric parameters on the efficiency and flow ratio. The results are presented in figures 8,9 and 10 .
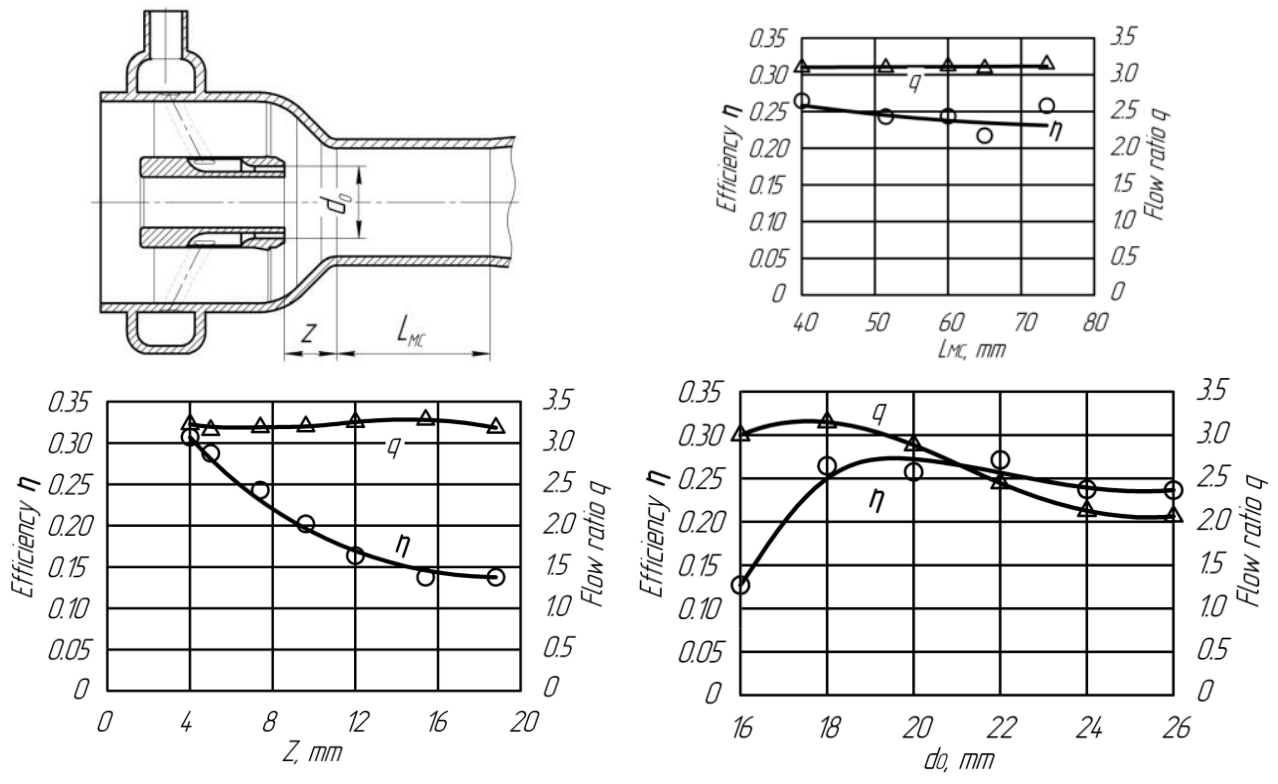

Fig. 8. Effect of geometric parameters $L_{M C}, z$ and $d_{0}$ on JP efficiency and flow ratio.
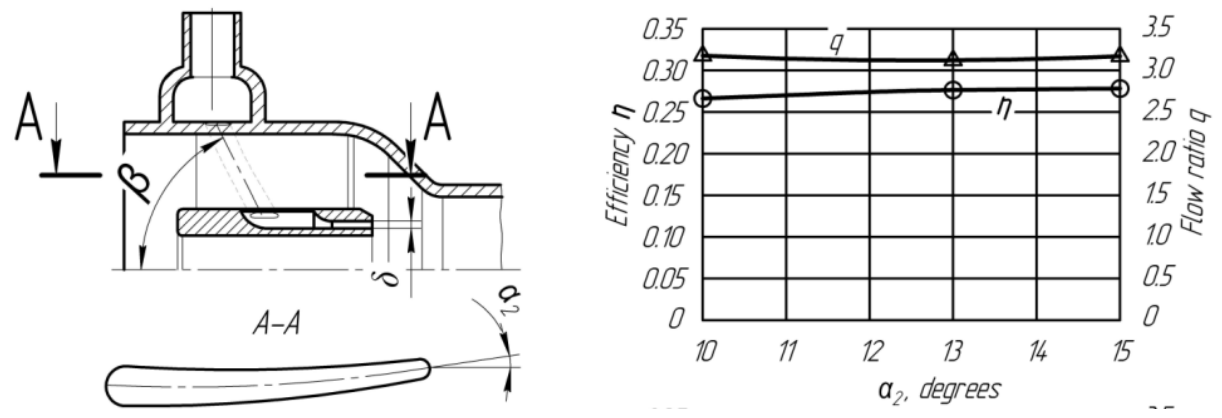

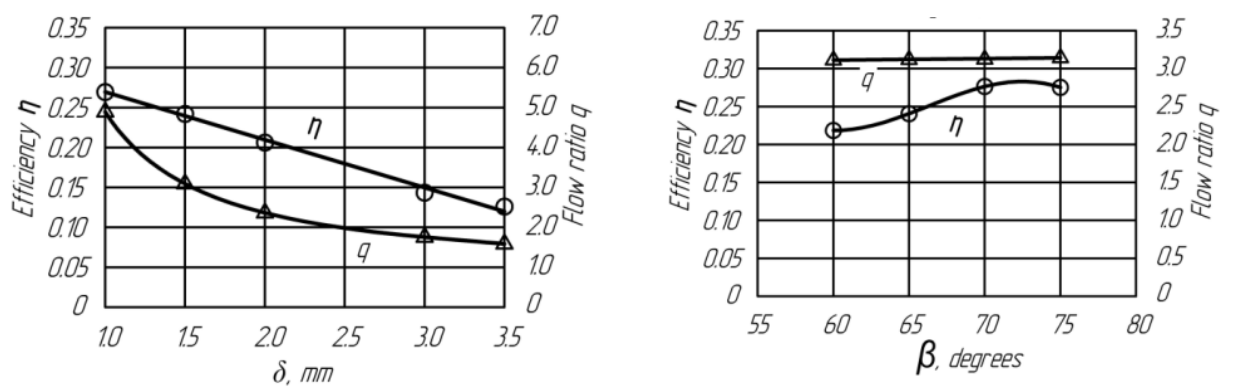

Fig. 9. Effect of geometric parameters $\alpha_{2}, \delta$ and $\beta$ on JP efficiency and flow ratio.
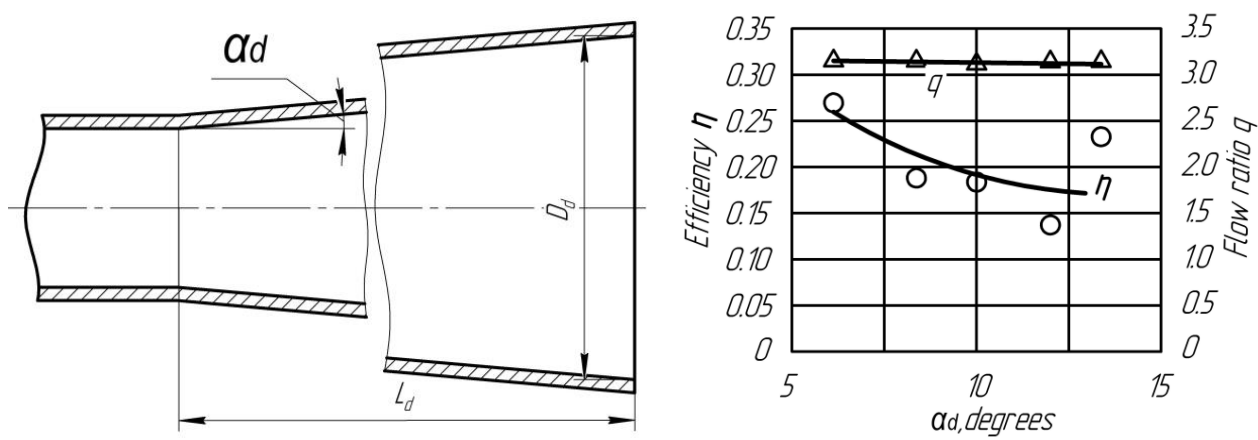

Fig. 10. Effect of geometric parameters $\alpha_{d}, l_{d}$ and $D_{d}$ on JP efficiency and flow ratio.

\section{Conclusion}

A possible implementation of a double surface jet pump with flows swirl (driving and suction) is proposed. The swirling of the driving flow in the proposed design is performed using tangential channels.

In JP with a flow swirl, the following positive aspects can be distinguished:

- increased efficiency compared to existing JP designs [10, 11];

- increased coefficient of ejection. This allows to obtain pumps for large flows for the same external energy parameters;

- reduced dimensions compared to existing JP designs;

- increased zone of operation.

Among the shortcomings of the JP with flow swirl, manufacturing complication can be noted due to the presence of a guide apparatus and tangential channels.

\section{References}

1. G. N. Abramovich, Theory of Turbulence (Moscow: Ekolit), 720 (2011)

2. I. S. Seitasanov, Study of hydraulic elevators with tangential suction medium inlet used in hydraulic engineering and land reclamation PhD Thesis, Taraz, 1999

3. R. B. Ahmedov, Swirling flows aerodynamics (Moscow: Energy), 240 (1977)

4. E. Y. Sokolov and N. M. Zinger, Jet pumps (Moscow: Energoatomizdat), 352 (1989) 
5. V. V. Aleksandrov, Improving the efficiency of reclamation pumping stations by introducing ejection into the suction and pressure pipelines of centrifugal pumps $P h D$ Thesis, Volgograd national agricultural university, Volgograd, 2012

6. R. Yapici and Aldas K, Optimization of water jet pumps using numerical simulation Journal of Power and Energy, 227(4), 2013, pp 438-449

7. A. H. Hammound, Effect of design and operational parameters on jet pump performance Proceedings of the 4th WSEAS International Conference on Fluid Mechanics and Aerodynamics, Elounda, Greece, 2006, pp 245-252

8. S. A. Tarasiyanc, Recommendations on choosing the optimal geometric dimensions of annular nozzles of jet pumps with a double surface working jet Online electronic journal of the Kuban State Agrarian University 75, 2012, pp 240-249

9. U. U. Zuev, The basics of creating competitive technology and developing effective solutions (Moscow: MPEI Publishing House), 2006

10. X. Yang, Long X, Kang Y and Xiao L. Application of constant rate of velocity or pressure change method to improve annular jet pump performance International Journal of Fluid Machinery and Systems 6, 2013, 137-43

11. A. Husain, Sonawat A, Mohan S and Samad A, Energy efficient design of a jet pump by ensemble of surrogates and evolutionary approach International Journal of Fluid Machinery and Systems 9, 2016, 265-76 